\title{
Cystic breast lesions by conventional ultrasonography: sonographic subtype-pathologic correlation and BI-RADS Assessment
}

\author{
Man Chen ${ }^{1,2}$, Wei Wei Zhan², Wen Ping Wang ${ }^{1}$
}

\author{
${ }^{1}$ Department of Diagnostic Ultrasound, Zhong Shan Hospital, Fu Dan University \\ School of Medicine, Shanghai, China \\ 2Department of Diagnostic Ultrasound, Rui Jin Hospital, Shanghai Jiao Tong University \\ School of Medicine, Shanghai, China
}

Submitted: 15 June 2011

Accepted: 12 December 2011

Arch Med Sci 2014; 10, 1: 76-83

DOI: 10.5114/aoms.2014.40734

Copyright $\odot 2014$ Termedia \& Banach

\section{Abstract}

Introduction: Appropriate categorization is very important because the clinical management of each subtype of cystic breast lesions (CBLs) differs. The purpose was to evaluate the sonographic subtype-pathologic correlation, and to identify the effectiveness of the Breast Imaging Reporting and Data System (BI-RADS)-ultrasound (US) for differentiation of benign and malignant CBLS.

Material and methods: A database from December 1, 2007 and November 30, 2009 was identified in the Department of Diagnostic Ultrasound, Rui Jin Hospital, School of Medicine, Shanghai Jiao Tong University, China. Those patients with palpable or clinical symptomatic breast masses were associated with a cystic component in lesions on breast US. All patients underwent a subsequent fine-needle/core-needle biopsy or surgical excision. The sonographic findings were analyzed according to the BI-RADS-US, and were categorized by two different methods of subtype categorization compared with the pathologic results.

Results: Ninety-nine breast cystic lesions in 83 women were included, among whom 16 patients were identified with bilateral cystic lesions. The total malignancy rate of CBLs was $14.1 \%$ (95\% confidence interval $7.3-21.0 \%$ ). Among 99 CBLs, 14 malignant lesions were associated with sonographic appearances of complex cystic lesions, while the remaining subtypes were benign. Shape, margin, echo pattern, orientation, calcification, and vascularity were statistically significantly different between the benign and malignant lesions $(p=0.010, p=0.004, p<0.001, p<0.001, p=0.036$, and $p<0.001$, respectively) (degrees of freedom $=1$ ).

Conclusions: By comparison of the two different methods of subtype categorization of CBLs, the appropriate 5-variety classification should be suggested. The BI-RADS-US was useful for differentiating benign from malignant cystic lesions.

Key words: breast, ultrasonography, subtype, pathology, Breast Imaging Reporting and Data System.

\section{Introduction}

Ultrasonography (US) has become an accepted modality advocated by many as a supplemental method of breast imaging, especially for differ-

\author{
Corresponding author: \\ Wen Ping Wang MD \\ Department \\ of Diagnostic Ultrasound \\ Zhong Shan Hospital \\ Fu Dan University \\ School of Medicine \\ No. 180 Fen Lin Road \\ 200032 Shanghai, China \\ Phone: +8602164041990 \\ E-mail: \\ maggiech1221@126.com
}


entiating cysts from solid masses [1-3], particularly in women with dense breasts [4-6].

In recent years, the first edition of the Breast Imaging Reporting and Data System (BI-RADS) for US (BI-RADS-US) has been developed by the American College of Radiology (ACR) to improve consistent feature analysis, interpretation, and reporting [7]. Previous studies indicated that the BIRADS lexicon was useful for differentiating benign from malignant solid masses [8, 9]. Gokalp et al. [9] reported that the sensitivity, specificity, positive predictive value (PPV) and negative predictive value (NPV) of BI-RADS-US and power Doppler US in the diagnosis of malignant solid lesions were $100 \%, 58.2 \%, 62.9 \%, 100 \%$ and $71.8 \%, 81.8 \%$, $73.7 \%, 80.4 \%$, respectively.

Cystic breast lesions (CBLs) comprise a wide spectrum of variable disease entities including benign and malignant breast diseases [10, 11]. In 2003 the ACR established the BI-RADS-US in different varieties of CBLs, including simple cyst, cluster cyst, complicated cyst, and complex cyst [7]. Given that patients with CBLs may have varied sonographic manifestation, classification of CBLS merits discussion. Appropriate categorization of CBLs is very important for general radiologists because the clinical practice in the management of each subtype differs. Several studies have assessed the classification of CBLs [10, 12-15]. The 3 -variety classification represented a more simple method and concentrates on 3 frequently encountered categories (simple, complicated and complex cysts) $[12,13]$. However, in accordance with Chang et al. [16], the CBLs were classified into 6 varieties as follows: simple cysts, clustered cysts, cysts with thin septa, complicated cysts, cystic lesions with a thick wall/septa or nodules, and complex solid and cystic masses.

To our knowledge, there is little in the literature comparing the two different methods of subtype categorization of CBLs, and describing the US features and final assessments using the BI-RADS lexicon. The CBLS with US appearance offer useful examples of lesions appropriate for the BI-RADS category and may increase the consistency and reproducibility of US imaging of these lesions.

The purpose of this study was to evaluate the sonographic subtype-pathologic correlation of CBLs, and to identify the effectiveness of the BI-RADS-US for differentiation of benign and malignant cystic lesions to provide an appropriate categorization of CBLs for assessment and management recommendations.

\section{Material and methods}

A database from December 1, 2007 and November 30, 2009 was identified in the Department of Diagnostic Ultrasound, Rui Jin Hospital, School of
Medicine, Shanghai Jiao Tong University, China. Our database is password protected. Recording and reviewing the results of these examinations and the results of subsequent imaging, clinical, and pathologic follow-up was approved by our local ethics committee. Patients with palpable or clinical symptomatic breast masses with US cystic component(s) that had fine-needle/core-needle biopsy or surgical excision were included in the study. Patients without a cystic component at breast US, or not undergoing biopsy, were excluded.

US-guided fine-needle aspiration alone was performed in 64 lesions from which fluid was withdrawn by using an 18- or 20-gauge needle and sent for cytologic examination. US-guided core needle biopsy was performed in 35 lesions with a 14-gauge automated biopsy gun (Monopty; Bard, Covington, Ga), and excision surgery was performed in 30 lesions. The pathological examiner was blinded to the results of clinical findings and/or US findings.

Bilateral whole-breast conventional breast sonography was performed using a Logiq 9 scanner (GE Medical Systems, Milwaukee, Wis) equipped with a L14-9 MHz transducer, and a Mylab 90 (Esaote, Genoa, Italy) ultrasound machine equipped with a L13-4 MHz transducer. Images were acquired in both radial and transverse planes. Additional gray-scale images were obtained in some cases to better show the lesion. All the sonographers have received extensive training and clinical experience in breast US and in the first edition of the BI-RADS-US, and the examiner knew the results of the clinical examination, without knowing the pathologic results. In patients with multiple lesions, assessment of category and management were based on the highest-category lesion. Moreover, if a patient had more than one lesion in the same category, we evaluated the largest/most dominant lesion.

The sonographic findings were analyzed by one radiologist with 5 years of experience in performing and interpreting breast US from a picture archiving and communications system (PACS) according to the BI-RADS-US [7], without knowledge of physical examination findings or pathological results. The cystic lesions of the breast were (categorized by two different methods $[12,13,16]$, into 3 varieties) as follows: type 1 ('simple cyst') was selected if the shape of the lesion was round, oval or gently lobulated, the margin was circumscribed, the echogenicity (echo pattern) was an echoic and there was acoustic enhancement; type 2 ('complicated cyst') was defined as cysts with homogeneous low-level internal echoes throughout a cystic lesion that had all the other features of a simple cyst [7], including cystic lesions containing fluid-debris levels or floating echogenic 
debris; type 3 ('complex cyst') as cysts with thick walls, thick septa, intracystic masses, or other discrete solid components; and 6 varieties (types I$\mathrm{VI}$ ) as follows: type I ('simple cyst') was defined as mentioned above; type II ('clustered cysts') indicated clustered anechoic cysts with no discrete solid components; type III ('cysts with thin septa') was defined as cysts within septa of less than $0.5 \mathrm{~mm}$ in thickness; type IV ('complicated cyst') was defined as mentioned above; type $V$ ('cystic lesions with a thick wall/septa or nodules') as cystic masses with thick septa $(\geq 0.5 \mathrm{~mm})$ or a thick wall ( $\geq 0.5 \mathrm{~mm}$ ) or mixed cystic and solid masses with at least a 50\% cystic component; type VI ('complex solid and cystic masses') was considered as primarily solid masses with eccentric cystic foci.

\section{Statistical analysis}

The SPSS version 16.0 statistical package for Windows (SPSS Inc, Chicago, IL) was used for statistical analysis. The lesion was characterized using BI-RADS ultrasound descriptors of mass margin (circumscribed, or not circumscribed (indistinct, microlobulated, angular or speculated)), shape (regular (oval or round) or irregular), orientation (parallel or not parallel to the skin), matrix echogenicity (complex or not complex (anechoic, hypoechoic, isoechoic, or hyperechoic)), posterior acoustic feature (absent or present (enhancement, shadowing or combined pattern)), calcification (present or absent), and vascularity (present or absent) [7]. The $t$ test was applied to determine whether the patients' age and the maximum di- ameter of the tumor were different in the benign and malignant groups. Fisher's exact test was performed to determine whether the lesion descriptors were different in the benign and malignant groups. For these tests, a $p$ value less than 0.05 was considered statistically significant. The diagnostic indices including odds ratio (OR) (95\% confidence interval $(95 \% \mathrm{CI}))$, sensitivity, specificity, positive and negative predictive value (PPV and NPV), and the receiver operating characteristic (ROC) curve were determined with exact 95\% confidence intervals.

\section{Results}

Ninety-nine breast cystic lesions in 83 women were included, among whom 16 patients were identified with bilateral cystic lesions. The mean age of patients was 51.47 years, ranging from 26 to 81 years (95\% Cl: $48.72-54.22$ years). The mean diameter of the lesions was $18.64 \mathrm{~mm}$, ranging from $3 \mathrm{~mm}$ to $47 \mathrm{~mm}$ (95\% Cl: $16.45-20.83 \mathrm{~mm}$ ).

The rate of malignant cystic lesions in each type is shown in Table I (Figures 1-6). Six remaining lesions were classified as 'others'. Among 99 CBLs, 14 malignant lesions were associated with sonographic appearance of type 3 in 3 varieties, type $\mathrm{V}$ and type $\mathrm{VI}$ in 6 varieties, while the other subtypes (simple cyst, clustered cysts, complicated cysts, and cysts with thin septa) were all benign. Malignant lesions included invasive ductal carcinoma $(n=4)$, ductal carcinoma in situ $(n=3)$, intraductal papillary carcinoma $(n=3)$, mucinous carcinoma $(n=2)$, lobular carcinoma in situ $(n=1)$,

Table I. Rates of malignancy in each subtype of cystic breast lesions

\begin{tabular}{|c|c|c|c|c|c|c|}
\hline Subtype & $n$ & Benign & Malignancy & Rate of malignancy (\%) & $\mathrm{d} f$ & Value of $p$ \\
\hline Three varieties & & & & & 3 & $<0.001$ \\
\hline Type 1 & 40 & 40 & 0 & 0.0 & & \\
\hline Type 2 & 18 & 18 & 0 & 0.0 & & \\
\hline Type 3 & 35 & 21 & 14 & $40.0(23.8-56.2)$ & & \\
\hline Others & 6 & 6 & 0 & 0.0 & & \\
\hline Total & 99 & 85 & 14 & $14.1(7.3-21.0)$ & & \\
\hline Six varieties & & & & & 5 & $<0.001$ \\
\hline Type I & 40 & 40 & 0 & 0.0 & & \\
\hline Type II & 2 & 2 & 0 & 0.0 & & \\
\hline Type III & 4 & 4 & 0 & 0.0 & & \\
\hline Type IV & 18 & 18 & 0 & 0.0 & & \\
\hline Type V & 15 & 11 & 4 & $26.7(4.3-49.0)$ & & \\
\hline Type VI & 20 & 10 & 10 & $50.0(28.1-71.9)$ & & \\
\hline Total & 99 & 85 & 14 & $14.1(7.3-21.0)$ & & \\
\hline
\end{tabular}

$n$-sample size of calculation, $d f$-degrees of freedom. 


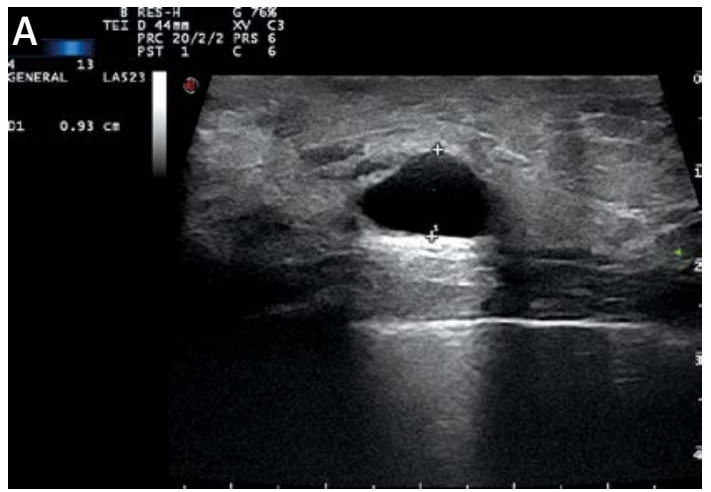

B

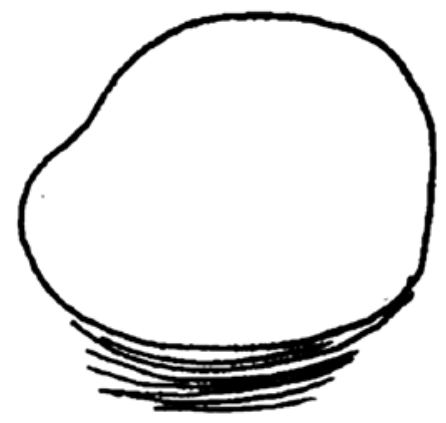

Figure 1. A - Sonogram of simple cyst with circumscribed margins, posterior acoustical enhancement, and no internal echoes. B - Schematic depiction of simple cyst

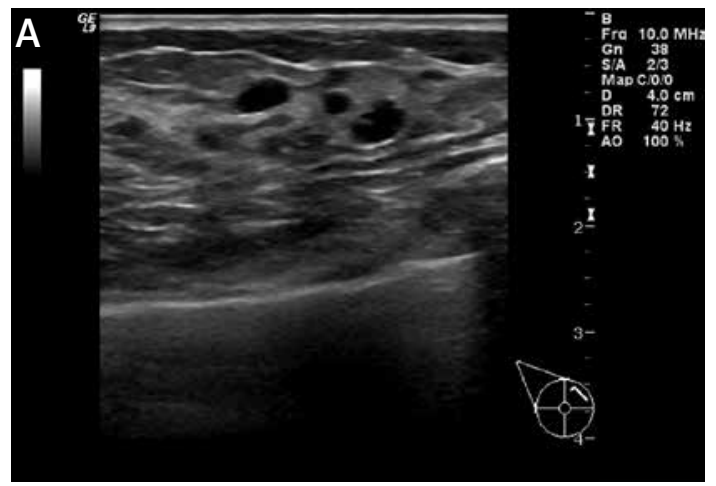

B
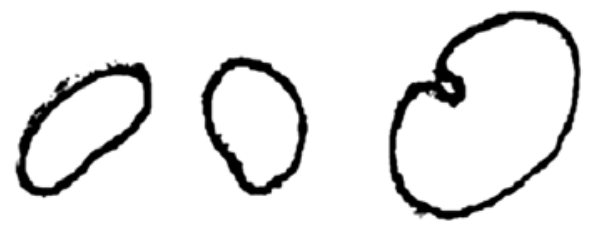

Figure 2. A - Sonogram of clustered cysts. B - Schematic depiction of clustered cysts

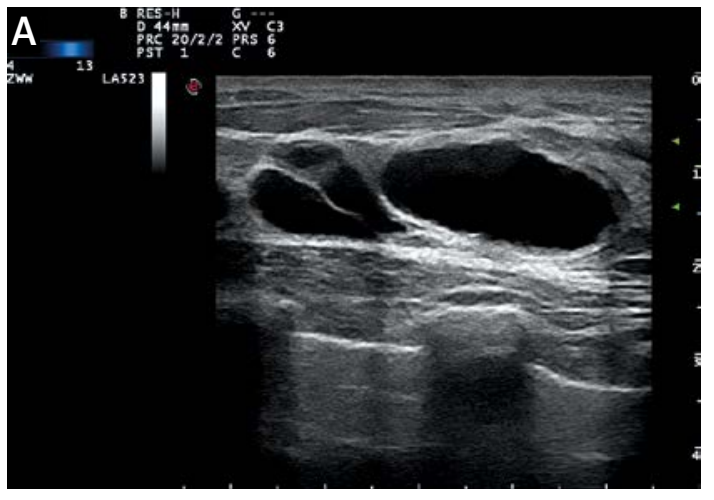

B

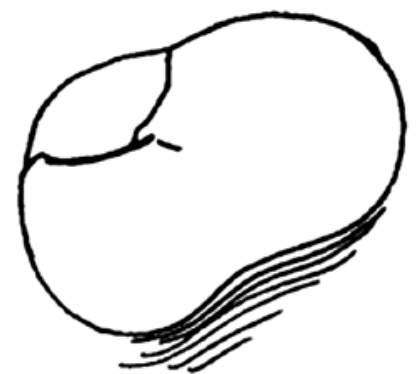

Figure 3. A - Sonogram of cyst with thin septa of less than $0.5 \mathrm{~mm}$ in thickness. B - Schematic depiction of cyst with thin septa of less than $0.5 \mathrm{~mm}$ in thickness

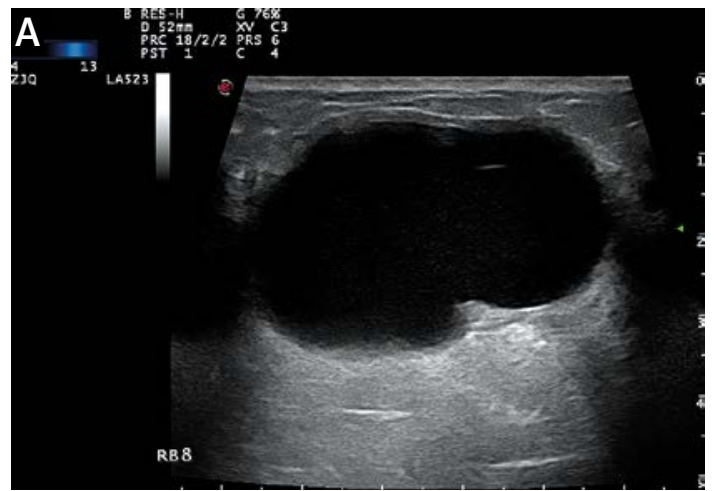

B

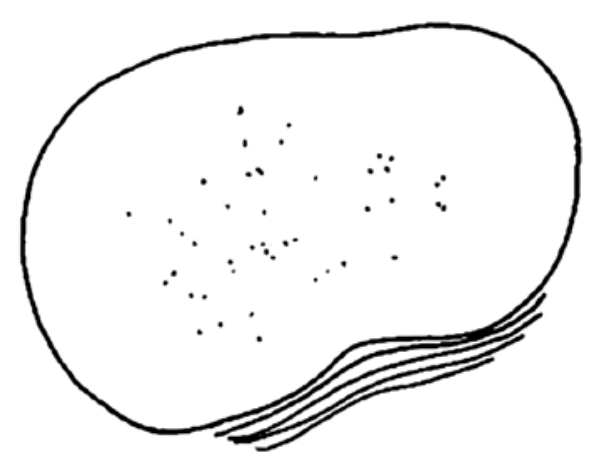

Figure 4. A - Sonogram of complicated cyst with circumscribed margins, posterior acoustical enhancement, and low-level internal echoes. B - Schematic depiction of complicated cyst 


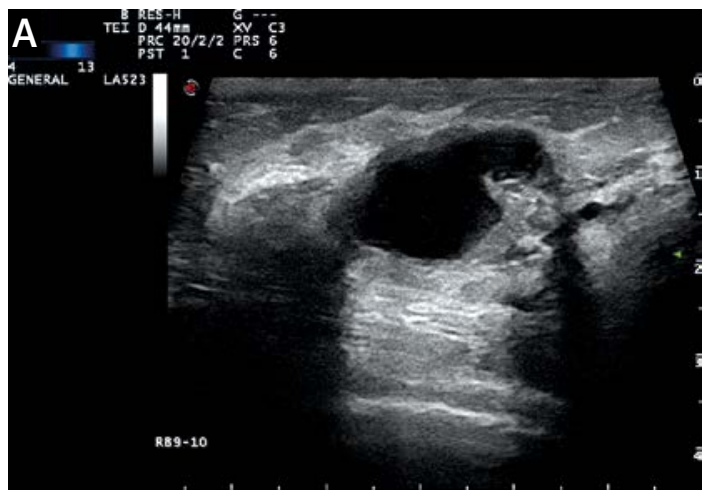

\section{B}

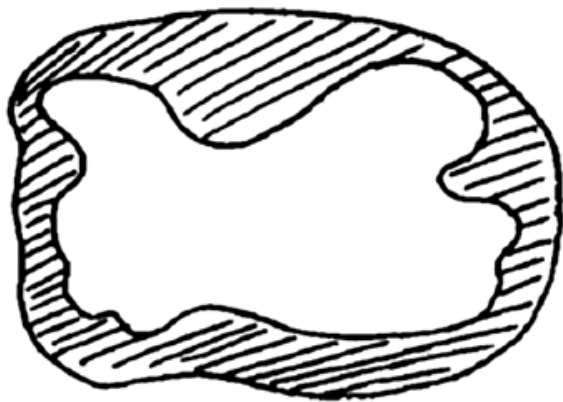

Figure 5. A - Sonogram of complex cystic lesion with septa or a wall greater than $0.5 \mathrm{~mm}$ in thickness. B - Schematic depiction of complex cystic lesion with septa or a wall greater than $0.5 \mathrm{~mm}$ in thickness

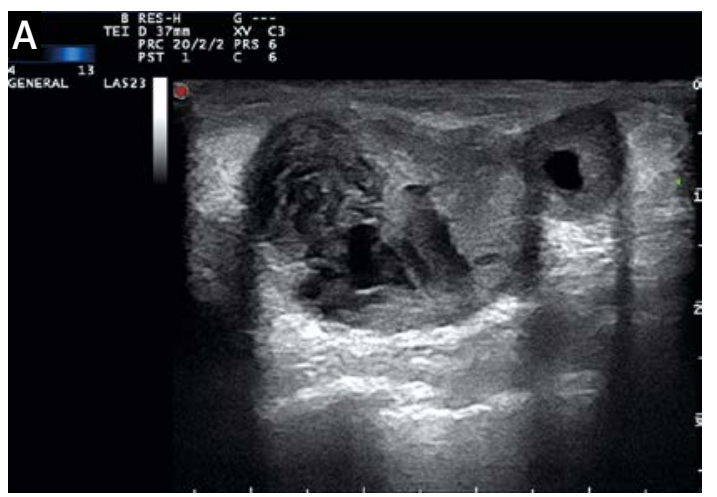

B

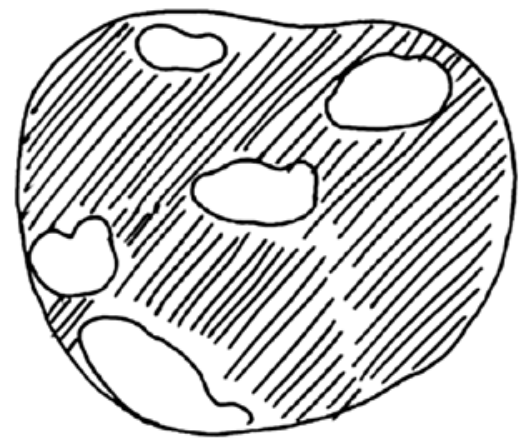

Figure 6. A - Sonogram of complex xystic lesion presented with complex solid and cystic masses. B - Schematic depiction of complex cystic lesion presented with complex solid and cystic masses

and medullary carcinoma $(n=1)$. The pathologic diagnoses of the remaining 83 benign lesions were 5 cysts, 10 papillomas, 54 fibrocystic changes, 9 cases of ductal ectasia, 5 cases of chronic inflammation, and 2 cases of fat necrosis.

Differences in the predominant shape, margin, echo pattern, orientation, calcification, and vascularity were statistically significant between the benign and malignant lesions in Table II, whereas the posterior acoustic feature did not show a significant difference. The diagnostic indices of sonographic descriptors according to the BI-RADS-US lexicon between benign and malignant CBLS, including odds ratio (OR) (95\% confidence interval $(95 \% \mathrm{CI}))$, sensitivity, specificity, PPV and NPV, are shown in Table II. The area under the curve (AUC) obtained by using a receiver operating characteristic curve (ROC) analysis to assess diagnostic performance is shown in Figure 7.

\section{Discussion}

Cystic breast lesions represent a composite of clinical and pathological diagnoses including developmental abnormalities, inflammatory lesions, epithelial and stromal proliferations, and neoplasms. Our study is consistent with several studies and has confirmed that cysts in women with the sonographic appearances of simple cysts, clustered cysts, and cysts with thin septa have no malignant potential $[7,10,12,16]$. Therefore, further diagnostic evaluation such as aspiration or biopsy are not necessary, unless the patient is symptomatic.

Previous reports mentioned that complicated cysts are likely to be benign $[10,12,16]$. The results from our study indicated that none of 18 complicated cysts proved to be malignant, consistent with previous studies. If the complicated cysts are clinically or mammographically new or are symptomatic, aspiration should be attempted $[12,17]$. In our series, the total malignancy rate of cystic breast lesions was $14.1 \%$ (14/99), slightly higher than the $12 \%(18 / 150)$ reported by Berg et al. [10] All these 14 malignant lesions were associated with sonographic appearances of complex cystic lesions, while the remaining subtypes were benign.

Complex cysts were the most common varieties associated with breast cancer compared to women with other varieties of CBLs. The term 'complex cyst', defined as cysts with thick walls, thick septa, or mixed solid and cystic components, distinguished from the other types of CBLs [7, 10, $11,16]$, is not a pathological classification; it is 


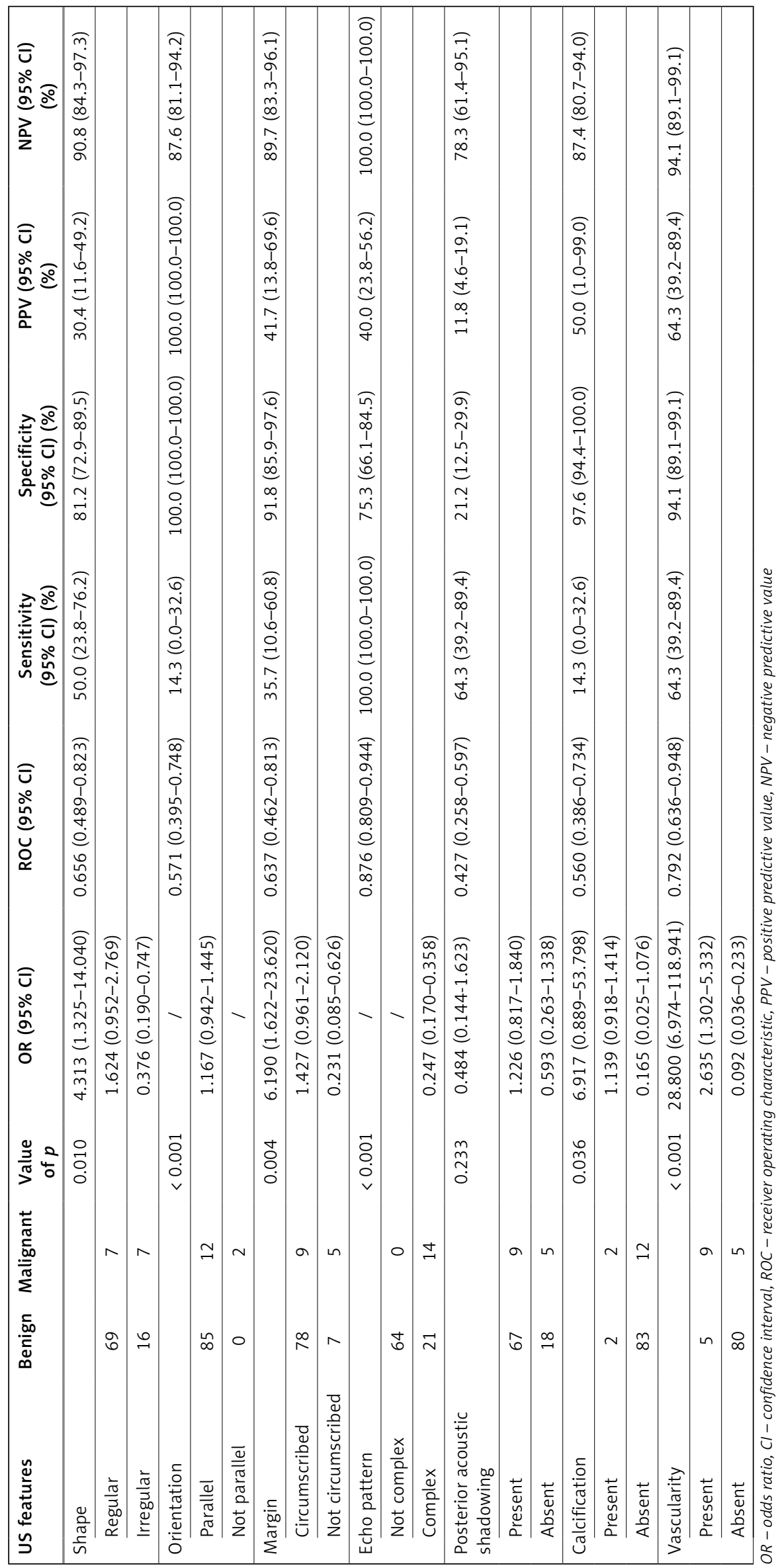




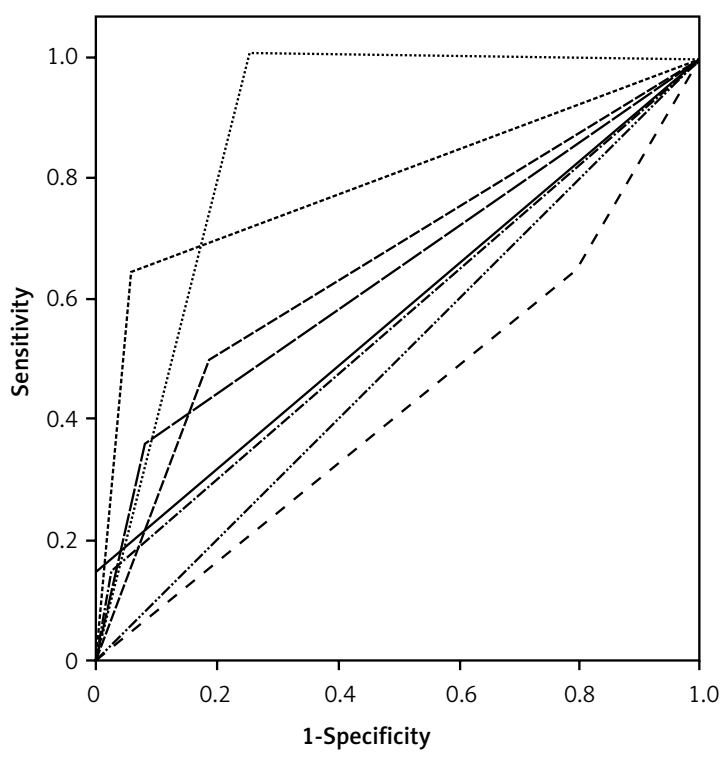

Source of the curve

$$
\begin{array}{ll}
\text {-- Margin } & --- \text { Posterior acoustic feature } \\
\text {----. Shape } & - \text { Orientation } \\
\text {-...-... Echo pattern } & \text {-.-- Calcification } \\
\text {-.-.-.. Vascularity in lesion } & \text {-.-.-. Reference line }
\end{array}
$$

Figure 7. The area under the Roc curve for diagnosis of malignant cystic breast lesions by sonographic descriptors according to the BI-RADS-US lexicon

a sonographic diagnosis with variations in definition and classification. Previous reports confirmed that complex cystic breast lesions encompass a wide range of pathologic entities, including benign, atypical (high-risk), and malignant lesions. Common benign diagnoses of complex cystic breast masses include fibrocystic changes, intraductal or intracystic papilloma, and fibroadenoma. Fibrocystic changes include adenosis, sclerosing adenosis, apocrine metaplasia, and ductal ectasia. Common malignant results include ductal carcinoma in situ (DCIS), intracystic carcinoma, and infiltrating ductal carcinoma [10, 12, 13, 16, 18, 19].

Furthermore, concerning complex cystic breast lesions associated with a significantly higher rate of malignancy, we recommend that the term 'complex cystic breast lesions' may be more suitable than 'complex cyst' in clinical use. Therefore, the appropriate term 'cystic breast lesions' (CBLs) should be suggested. US-guided percutaneous biopsy is an effective method for diagnosing and guiding the management of complex cystic masses.

Based on our experience, the 3-variety method excluded 4 lesions with thin septa and 2 lesions with the sonographic appearances matched with clustered cysts, although those 6 cystic lesions were proven to be benign. To the best of our knowledge, differentiation between type $\mathrm{V}$ and type $\mathrm{VI}$ varieties of complex cysts may be unnecessary because all these complex cysts require biopsy. Therefore, the appropriate 5 -variety classification for CBLs should be suggested, including simple cysts, clustered cysts, complicated cysts, cysts with thin septa, and complex cystic lesions.

In our study, the sonographic descriptors of CBLs were statistically significant variables associated with malignancy according to the BI-RADSUS, including shape, margin, echo pattern, orientation, calcification, and vascularity. Furthermore, regular shape, parallel orientation, circumscribed margin, non-complex echo pattern, absence of posterior acoustic feature (no posterior acoustic features), calcification, and vascularity of lesions showed high predictive value for benign lesions. Not parallel to skin and presence of vascularity of lesions showed high predictive value for malignant cystic lesions. Moreover, a complex echo pattern is more valuable in terms of malignancy, with the area under the ROC curve 0.876 , sensitivity $100 \%$, and specificity $75.3 \%$.

We recognize that our study had some weaknesses. In particular, although 99 cystic breast lesions were included in the study, the sample was small and some subgroups contained few cases, which limited the statistical power of the analysis. Additional investigations of larger numbers of lesions are necessary. Second, because our study was retrospective and selection bias was introduced by limiting entry to patients who had undergone a US examination, it did not represent the general population. In addition, although all the sonographers in this study have received extensive training and clinical experience in the breast ultrasound and the first edition of the BI-RADSUS, interobserver variability remains a concern. Taking this into consideration, all the sonographic findings were analyzed by one radiologist with 5 years of experience at performing and interpreting breast US from PACS, without knowledge of physical examination findings or pathological results.

In conclusion, the appropriate 5-variety classification for CBLs should be suggested, including simple cysts, clustered cysts, complicated cysts, cysts with thin septa, and complex cystic lesions. Complex echo pattern, irregular shape, nonparallel orientation, and noncircumscribed margin, associated with microcalcification or vascularity, are useful US predictors for subsets of probable malignancies in patients with cystic breast lesions. Clearly, this conclusion will be strengthened by further analyses of larger series and with prospective consecutive case series.

\section{References}

1. Bassett LW, Kimme-Smith C. Breast sonography. Am J Roentgenol 1991; 156: 449-55.

2. Jackson VP. The role of US in breast imaging. Radiology 1990; 177: 305-11. 
3. Parker SH, Jobe WE, Dennis MA, et al. US-guided automated large core breast biopsy. Radiology 1993; 187: 507-11.

4. Nothacker M, Duda V, Hahn M, et al. Early detection of breast cancer: benefits and risks of supplemental breast ultrasound in asymptomatic women with mammographically dense breast tissue. A systematic review. BMC Cancer 2009; 9: 335.

5. Kolb TM, Lichy J, Newhouse JH. Comparison of the performance of screening mammography, physical exam ination, and breast US and evaluation of factors that influence them: an analysis of 27,825 patient evaluations. Radiology 2002; 225: 165-75.

6. Buchberger W, DeKoekkoek-Doll P, Springer P, Obrist P, Dünser $M$. Incidental findings on sonography of the breast: clinical significance and diagnostic workup. AJR Am J Roentgenol 1999; 173: 921-7.

7. Mendelson EB, Baum JK, Berg W, Merritt C, Rubin E. Breast Imaging Reporting and Data System BIRADS: ultrasound. Reston VA: American College of Radiology 2003.

8. Hong AS, Rosen EL, Soo MS, Baker JA. BI-RADS for sonography: positive and negative predictive values of sonographic features. AJR Am J Roentgenol 2005; 184: 1260-5.

9. Gokalp G, Topal U, Kizilkaya E. Power Doppler sonography: anything to add to BI-RADS US in solid breast masses? Eur J Radiol 2009; 70: 77-85.

10. Berg WA, Campassi Cl, Ioffe OB. Cystic lesions of the breast: sonographic-pathologic correlation. Radiology 2003; 227: 183-91.

11. Venta LA, Dudiak CM, Salomon CG, et al. Sonographic evaluation of the breast. Radiographics 1994; 14: 29-50.

12. Huff JG. The sonographic findings and differing clinical implications of simple, complicated, and complex breast cysts. J Natl Compr Canc Netw 2009; 7: 1101-5.

13. Doshi DJ, March DE, Crisi GM, Coughlin BF. Complex cystic breast masses: diagnostic approach and imaging-pathologic correlation. Radiographics 2007; 27: S53-64.

14. Hashemi-Meshkini A, Keshavarz K, Gharibnaseri Z, et al. Cost-effectiveness analysis review of exemestane in the treatment of primary and advanced breast cancer. Arch Med Sci 2013; 9: 472-8.

15. Badowska-Kozakiewicz AM, Sobol M, Patera J, Kozłowski W. Immunohistochemical evaluation of human epidermal growth factor receptor 2 and estrogen and progesterone receptors in invasive breast cancer in women. Arch Med Sci 2013; 9: 466-71.

16. Chang YW, Kwon KH, Goo DE, Choi DL, Lee HK, Yang SB. Sonographic differentiation of benign and malignant cystic lesions of the breast. J Ultrasound Med 2007; 26: 47-53.

17. Daly CP, Bailey JE, Klein KA, et al. Complicated breast cysts on sonography: is aspiration necessary to exclude malignancy? Acad Radiol 2008; 15: 610-7.

18. Tea MK, Grimm C, Fink-Retter A, et al. The validity of complex breast cysts after surgery. Am J Surg 2009; 197: 199-202

19. Hsu HH, Yu JC, Lee HS, et al. Complex cystic lesions of the breast on ultrasonography: feature analysis and $\mathrm{BI}-$ RADS assessment. Eur J Radiol 2011; 79: 73-9. 